\section{Splitting the task: Ubp8 and Ubp10 deubiquitinate different cellular pools of H2BK123}

\author{
Julia M. Schulze, ${ }^{1,2,6}$ Thomas Hentrich, 1,3,6 \\ Shima Nakanishi, ${ }^{4}$ Arvind Gupta, ${ }^{3}$ \\ Eldon Emberly, ${ }^{5}$ Ali Shilatifard, ${ }^{4,7}$ \\ and Michael S. Kobor ${ }^{1,2,7,8}$
}

\begin{abstract}
${ }^{1}$ Centre for Molecular Medicine and Therapeutics, Child and Family Research Institute, Vancouver, British Columbia V5Z 4H4, Canada; ${ }^{2}$ Department of Medical Genetics, ${ }^{3}$ Department of Computing Science, University of British Columbia, Vancouver, British Columbia V5Z 4H4, Canada; ${ }^{4}$ Stowers Institute for Medical Research, Kansas City, Missouri 64110, USA; ${ }^{5}$ Department of Physics, Simon Fraser University, Burnaby, British Columbia V5A 1S6, Canada
\end{abstract}

Monoubiquitination of H2BK123 (H2BK123ub), catalyzed by Rad6/Bre1, is a transient histone modification with roles in transcription and is essential for establishing H3K4 and H3K79 trimethylations (H3K4me3 and H3K79me3). Here, we investigated the chromatin network around H2BK123ub by examining its localization and cooccurrence with its dependent marks as well as the transcription elongation mark H3K36me3 across the genome of Saccharomyces cerevisiae. In yeast, $\mathrm{H} 2 \mathrm{BK} 123 \mathrm{ub}$ is removed by the deubiquitinases Ubp8 and Ubp10, but their genomic target regions remain to be determined. Genomewide maps of H2BK123ub in the absence of Ubp8 and Ubp10 revealed their distinct target loci, which were genomic sites enriched for H3K4me3 and H3K79me3, respectively. We propose an extended model of the H2BK123ub cross-talk by integrating existing relationships with the substrate specificities of Ubp8 and Ubp10 reported here.

Supplemental material is available for this article.

Received August 19, 2011; revised version accepted September 27,2011

In eukaryotic cells, chromatin packages DNA into the nucleus and affects various aspects of genome function. As the fundamental components of chromatin, histone proteins are subject to a variety of post-translational modifications, including methylation, monoubiquitination, and acetylation (Shilatifard 2006; Smith and Shilatifard 2010). In a process referred to as histone cross-talk, some histone modifications may trigger others, resulting in

[Keywords: chromatin; histone modifications; histone cross-talk; H2B monoubiquitination; deubiquitination]

${ }^{6}$ These authors contributed equally to this work.

${ }^{7}$ These authors contributed equally to this work.

${ }^{8}$ Corresponding author.

E-mail msk@cmmt.ubc.ca.

Article is online at http://www.genesdev.org/cgi/doi/10.1101/gad.177220.111. distinct and coordinated localization patterns that regulate processes such as transcription, DNA replication, and repair (Lee et al. 2010). An evolutionarily conserved regulatory component of the transcription cycle is the cross-talk between H2BK123 monoubiquitination (H2BK123ub) and $\mathrm{H} 3 \mathrm{~K} 4$ and $\mathrm{H} 3 \mathrm{~K} 79$ trimethylation $(\mathrm{H} 3 \mathrm{~K} 4 \mathrm{me} 3$ and H3K79me3), whereby H2BK123ub is essential to establish the trimethylation marks (Shilatifard 2006).

While previous studies revealed aspects of this crosstalk and identified major components of the associated enzymatic machinery, a comprehensive genome-wide understanding of H2BK123ub localization, including the co-occurrence with its dependent marks and the rules governing its removal, is still lacking. H2BK123ub is a transient histone mark, which is established by the Rad6/Bre1 ubiquitin ligase complex during transcription initiation and elongation (Shilatifard 2006). In Saccharomyces cerevisiae, H2BK123ub localizes to promoter and coding regions of $P M A 1, A D H 1$, and $P Y K 1$, as well as to GAL1 during gene induction (Dover et al. 2002; Henry et al. 2003; Wood et al. 2003; Kao et al. 2004; Xiao et al. 2005; Schulze et al. 2009), while in human cells it is enriched at transcribed regions of highly transcribed genes (Minsky et al. 2008). Improper monoubiquitination of H2B disturbs transcription elongation at GAL1 in $S$. cerevisiae (Shukla and Bhaumik 2007) and alters the localization of RNA polymerase II (RNAPII) and histones in coding regions of hem $2^{+}$and sod2 $2^{+}$in Schizosaccharomyces pombe (Tanny et al. 2007).

An integral part of the H2BK123ub cross-talk is the removal of H2BK123ub by the ubiquitin-specific proteases Ubp8 and Ubp10 (Henry et al. 2003; Emre et al. 2005). Ubp8 is a subunit of the Spt-Ada-Gcn5acetyltransferase (SAGA) complex, with the integrity of SAGA being required for Ubp8 deubiquitination activity (Henry et al. 2003). Ubp8 acts mainly at early steps of transcription and regulates the transition between initiation and elongation (Henry et al. 2003; Daniel et al. 2004). In contrast, Ubp10 (also known as Dot4) acts independently of SAGA and has been associated with telomeric silencing (Kahana and Gottschling 1999; Emre et al. 2005; Gardner et al. 2005; Smith and Shilatifard 2009). Based on these functional differences and bulk protein blotting results showing increased ubiquitin levels in the

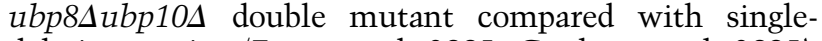
deletion strains (Emre et al. 2005; Gardner et al. 2005), we propose that Ubp 8 and Ubp10 have nonredundant roles in the H2BK123ub cross-talk and act in a site-specific manner.

To test this hypothesis, we comprehensively compare H2BK123ub levels in wild type with ubp8s and ubp10s mutants. Furthermore, we extend our previous study describing the colocalization of H2BK123ub with H3K79me3 (Schulze et al. 2009) by determining the co-occurrences of H2BK123ub with both its dependent modifications and the transcription elongation mark H3K36me3. We reveal that Ubp8 and Ubp10 have site-specific roles, with Ubp8 deubiquitinating $\mathrm{H} 2 \mathrm{BK} 123 \mathrm{ub}$ at $\mathrm{H} 3 \mathrm{~K} 4 \mathrm{me} 3$-marked regions and Ubp10 removing H2BK123ub at H3K79me3enriched sites. In addition, detailed analyses identified region-specific co-occurrences of $\mathrm{H} 2 \mathrm{BK} 123 \mathrm{ub}$ and $\mathrm{H} 3$ methylation marks, allowing us to propose a more complete model of the H2BK123ub cross-talk. 


\section{Results and Discussion}

Genome-wide distribution of H2BK123ub and H3 methylation marks with respect to gene length and transcriptional frequency

To define the chromatin network around $\mathrm{H} 2 \mathrm{BK} 123 \mathrm{ub}$ in S. cerevisiae, we mapped H2BK123ub; its dependent marks, H3K4me3 and H3K79me3; and the transcription elongation mark H3K36me3 using high-resolution tiling arrays. We also included H3K79me2 as a control for specificity. H2BK123ub and the $\mathrm{H} 3$ methylation marks were strongly enriched in genomic regions transcribed by RNAPII but mostly absent from other genomic features, such as telomeres, centromeres, the rRNA locus, ARSs, and tRNAs (Supplemental Table S1). Therefore, we focused our analysis on RNAPII transcripts and developed a compact yet comprehensive visualization approach (CHROMATRA) to assess the distribution of histone modifications across all transcripts at once while accounting for gene length and transcriptional frequency ( $\mathrm{T}$ Hentrich, JM Schulze, E Emberly, and MS Kobor, in prep.).

Our work extends existing studies on single genes to encompass the entire genome and finds H2BK123ub to predominantly cover coding sequences of genes as well as some promoters (Supplemental Fig. S1). Its dependent mark, H3K4me3, peaked sharply downstream from the transcription start site (TSS); H3K79me3 covered the body of mainly longer transcripts; H3K79me2 marked mainly shorter genes; and H3K36me3 was enriched beginning at the +3 nucleosome throughout the body of transcripts (Supplemental Fig. S1).

To examine the correlation between the modifications of the H2BK123ub network and transcriptional gene activity, all transcripts were grouped into five classes according to their transcriptional frequency (Holstege et al. 1998). H2BK123ub was present in all classes (Fig. 1A), and the relative occupancy was positively correlated with the transcriptional frequency of genes (Fig. 1B). H3K4me3 showed a trend similar to H2BK123ub but with higher enrichment levels (Fig. 1A,B) and, as recently described (Zhang et al. 2011), was enriched at almost all genes despite earlier proposals that it is limited to highly transcribed genes (Santos-Rosa et al. 2002; Ng et al. 2003; Liu et al. 2005). The most highly transcribed genes were instead specifically enriched for a combination of H2BK123ub, H3K4me3, and H3K36me3 (Fig. 1A). In contrast, enrichment levels of H3K79me3 and H3K79me2 strongly decreased for genes with higher transcriptional frequency (Fig. 1A), rendering the most actively transcribed genes devoid of these marks (Fig. 1B). Interestingly, the association of $\mathrm{H} 3 \mathrm{~K} 36 \mathrm{me} 3$ and transcriptional activity was nearly identical to that found for H2BK123ub (Fig. 1), supporting a role of H2BK123ub in transcriptional elongation.
H2BK123ub positively correlates and colocalizes with its dependent marks, H3K4me3 and H3K79me3, but also with H3K36me3

Consistent with its role as an upstream regulator, the H2BK123ub profile positively correlated with those of its dependent marks, H3K4me3 and H3K79me3, although the correlation was much stronger with $\mathrm{H} 3 \mathrm{~K} 79$ me3 $(r=0.67)$ than with H3K4me3 $(r=0.26)$ (Supplemental Fig. S2A). Supporting our earlier qualitative statement, H2BK123ub correlated positively with the elongation mark H3K36me3 $(r=0.63)$, consistent with the role of H2BK123ub in transcriptional elongation. To examine the relationship of H2BK123ub and H3 methylation in more detail, genes were partitioned into five segments (promoter, TSS-proximal, 5 ' coding sequence (CDS) mid-CDS, and 3'CDS), similar to an approach used previously (Liu et al. 2005), and the average enrichment score for each modification was calculated, hierarchically clustered, and visualized as a heat map (Supplemental Fig. S2B). Among several distinctive combinations of modification patterns, H2BK123ub strongly colocalized with H3K4me3 in the TSS-proximal segment, whereas H2BK123ub and H3K79me3, together with $\mathrm{H} 3 \mathrm{~K} 36 \mathrm{me} 3$, co-occurred predominantly in the mid-CDS segment (Supplemental Fig. S2B; Supplemental Table S2).

Although nearly all regions enriched for H2BK123ub were also marked by H3K4me3 or H3K79me3, not all 
sites enriched for $\mathrm{H} 3 \mathrm{~K} 4 \mathrm{me} 3$ and $\mathrm{H} 3 \mathrm{~K} 79 \mathrm{me} 3$ also carried H2BK123ub (Supplemental Fig. S2B). This observation might be attributable to the transient nature of $\mathrm{H} 2 \mathrm{BK} 123 \mathrm{ub}$ and its mode of removal by the deubiquitinases Ubp 8 and Ubp10, and likely explains the rather low rank correlation of $r=0.26$ between H2BK123ub and H3K4me3.

\section{Site-specific removal of H2BK123ub}

by its deubiquitinases, Ubp 8 and Ubp10

To determine the genomic regions that Ubp8 and Ubp10 act on, we mapped H2BK123ub across the genome in strains lacking either Ubp8 or Ubp10. As expected from studies based on bulk protein levels (Emre et al. 2005; Gardner et al. 2005), the number of H2BK123ub-enriched probes within transcripts strongly increased in both deletion strains from $24.8 \%$ in wild-type cells to $34.2 \%$ in the $u b p 8 \Delta$ strain and $32.9 \%$ in the ubp10s strain (Fig. 2A). Supporting our hypothesis that Ubp8 and Ubp10 act at distinct genomic loci, newly enriched probes for H2BK123ub were different between the two deletion strains, with $\sim 11 \%$ of all transcribed probes being uniquely deubiquitinated by Ubp 8 and $9 \%$ being uniquely deubiquitinated by Ubp 10 (Fig. 2A). To assess the location of ubiquitinated regions in both deletion strains, CHROMATRA was used to visualize the H2BK123ub distribution across all transcripts (Fig. 2B; Supplemental Fig. S3). In the ubp8s strain, H2BK123ub peaked downstream from the TSS, but was reduced in the body of transcripts compared with wild-type cells (Fig. 2B; Supplemental Fig. S3). In contrast, H2BK123ub localized to the coding sequence of mainly longer genes in the ubp10A strain (Fig. 2B; Supplemental Fig. S3). To better visualize the localization of newly enriched sites along the transcripts, we subtracted wild type from deletion profiles and color-coded positive-definite results (Fig. 2C). The resulting profiles clearly indicated a site-specific removal of H2BK123ub by Ubp8 in the

A
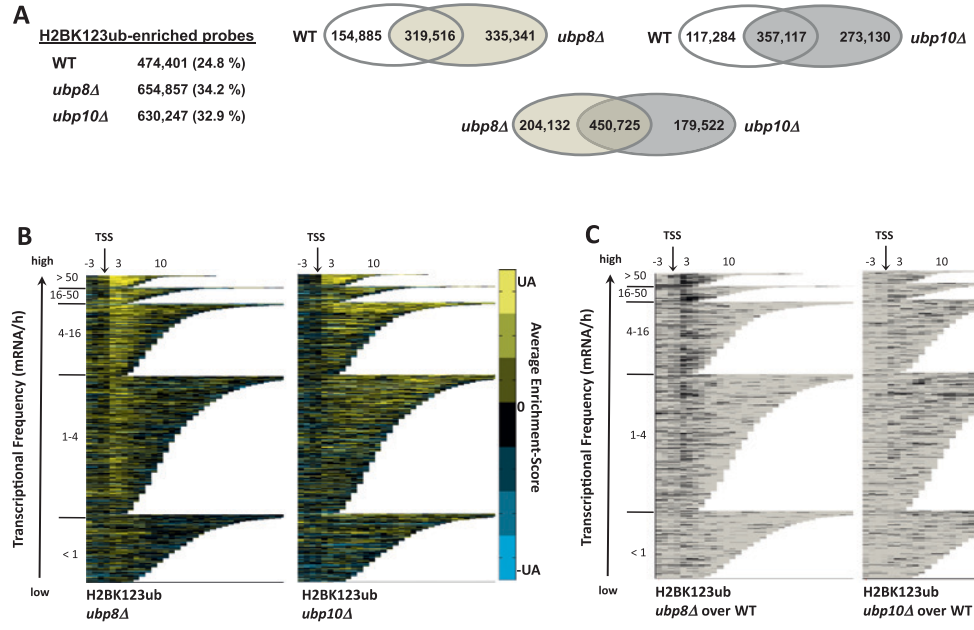

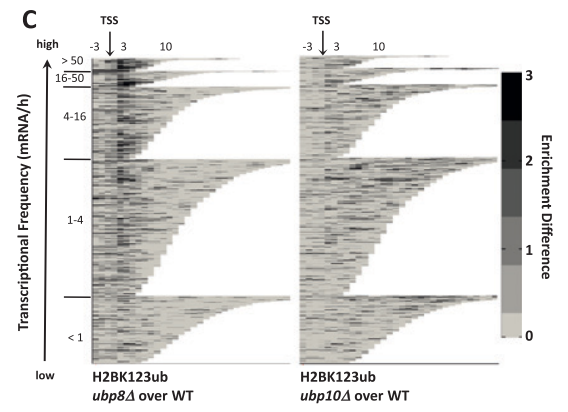

Figure 2. Site-specific removal of H2BK123 ubiquitin by Ubp8 and Ubp10. (A) Number of probes enriched for H2BK123ub within transcripts in wild-type as well as ubp8 and ubp10 deletion strains. Venn diagrams comparing the overlap of these probes between the different strains. (B) Distribution of H2BK123ub in wild-type as well as ubp8 and ubp10 deletion strains across all transcripts, sorted by their transcriptional frequencies. Calculations and plotting as in Figure 1A. $(C)$ Differences in the enrichment of H2BK123ub in ubp8 and ubp10 deletion strains. Enrichment scores for H2BK123ub in ubp8 and ubp10 deletion strains were subtracted from wild-type enrichment scores and only positive-definite results were color-coded. Average enrichment was calculated and transcripts were sorted as in Figure 1A.
TSS-proximal region and by Ubp10 in the gene-coding region.

By averaging the enrichment profiles of $\mathrm{H} 2 \mathrm{BK} 123 \mathrm{ub}$ similar to a previous approach (Mayer et al. 2010), we noticed that the H2BK123ub profile was very different $4 \mathrm{me} 3$ but comparable in its lateral distribution and overall shape with H3K79me3 (Fig. 3). Upon deletion the rank correlation between H2BK123ub and 0.45 in the ubp $8 \Delta$ strain. The ubp 10 deletion profile however, had relatively modest changes, although the degree of resemblance to $\mathrm{H} 3 \mathrm{~K} 79 \mathrm{me} 3$ further increased (Fig. 3). These data suggest that Ubp 8 acts primarily in ubiquitinates H2BK123 in the body of transcripts marked H3K79me3.

that in wild-type cells with both deubiquitinases present the measured $\mathrm{H} 2 \mathrm{~K} 123 \mathrm{ub}$ level reflects the least fraction captured by chromatin immunoprecip-

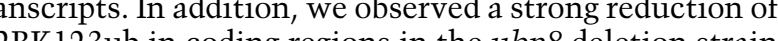
(Fig. 3). This might suggest that nonremoval of the ubiquitin tag at the 5'CDS caused by loss of Ubp8 hinders the transcriptional machinery to properly elongate and ubiquitinate downstream regions, thus resulting in strongly reduced ubiquitination of H2BK123 in the body transcripts. deubiquitinases and the H2BK123ub-dependent marks, examined H2BK123ub-enriched probes for co-occupancy with H3K4me3 or H3K79me3 in wild type, ubp8s, and ubp10s (Fig. 4A). While the number of H2BK123ubenriched probes co-occurring with H3K4me3 strongly increased in the ubp $8 \Delta$ strain, probes co-occurring with H3K79me3 mainly increased in the ubp10s strain (Fig. 4A). Since the observed changes occurred mostly in the TSS-proximal and mid-CDS regions, we analyzed these gene segments further and determined the overlap of deubiquitinated segments by Ubp 8 and Ubp10 with segments marked by H3K4me3 and H3K79me3 (Fig. 4B). Supporting our hypothesis, regions downstream from the TSS uniquely deubiquitinated by Ubp8 were mainly marked by $\mathrm{H} 3 \mathrm{~K} 4 \mathrm{me} 3$, while very few of these regions were solely enriched for $\mathrm{H} 3 \mathrm{~K} 79 \mathrm{me} 3$. In contrast, mid-CDS segments uniquely deubiquitinated by Ubp 10 were mainly marked by H3K79me3, with H3K4me3 being mostly absent at these sites (Fig. 4B).

Despite the distinct lateral localization of $\mathrm{H} 3 \mathrm{~K} 4 \mathrm{me} 3$ and $\mathrm{H} 3 \mathrm{~K} 79 \mathrm{me} 3$ along genes, they overlapped at the transition of TSS-proximal and mid-CDS (Figs. 1, 4). This suggested that both Ubp8 and Ubp10 might be active 

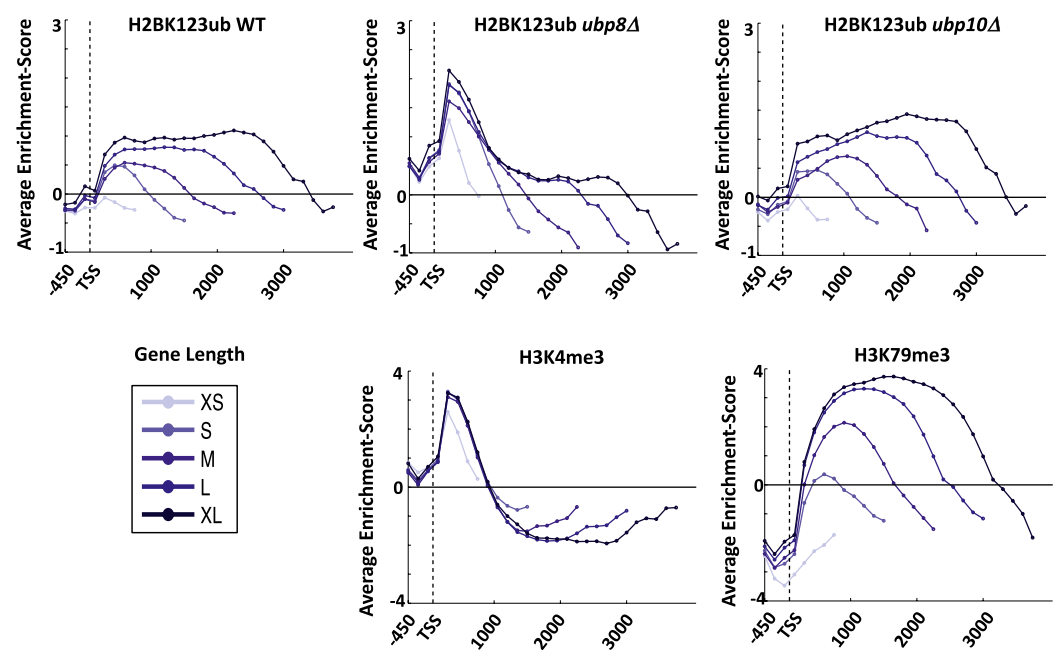

Figure 3. H2BK123ub profiles in ubp8 and ubp10 deletion strains resembled H3K4me3 and H3K79me3 profiles, respectively. All genes with known TSSs were divided into five length classes, and the average enrichment for H2BK123ub wild-type and ubp 8 and ubp10 deletion strains as well as $\mathrm{H} 3 \mathrm{~K} 4 \mathrm{me} 3$ and $\mathrm{H} 3 \mathrm{~K} 79 \mathrm{me} 3$ were plotted in 150-bp increments. The $\mathrm{H} 2 \mathrm{BK} 123 \mathrm{ub}$ profiles in the $u b p 8 \Delta$ and $u b p 10 \Delta$ strains resembled the averaged profiles of H3K4me3 and H3K79me3, respectively.

at these sites despite their otherwise exclusive target regions.

Circuitry of H2BK123ub and its dependent marks, H3K4me3 and H3K79me3

To further dissect the circuitry of H2BK123 ubiquitination/ deubiquitination and its dependent marks, we tested whether loss of Ubp8 or Ubp10 had any consequences on the genome-wide distribution of H3K4me3 and H3K79me3. Extending previous observations based on bulk protein blot analyses (Daniel et al. 2004; Gardner et al. 2005; Song and Ahn 2010), we found that the genome-wide distribution of H3K4me3 and H3K79me3 remained largely unchanged, and the site-specific location of these marks was unaffected upon loss of either Ubp8 or Ubp10 (Supplemental Fig. S4). These findings suggest a temporal relation in which the transient H2BK123ub mark triggers the establishment of the relatively stable H3K4me3 and H3K79me3 marks prior to its removal by Ubp 8 and Ubp10.

Integrating our findings with the current understanding of the H2BK123ub cross-talk, we propose the following model (Fig. 5). Initially, Rad6/Bre1 is recruited to promoters through interactions with transcriptional activators, catalyzing the monoubiquitination of H2BK123 (Smith and Shilatifard 2010). Together with the surrounding histone residues, H2BK123ub then provides a molecular "tag," attracting Set1/COMPASS, which subsequently trimethylates H3K4 (Lee et al. 2007; Kim et al. 2009; Zheng et al. 2010). Eventually, Ubp8 removes the bulky H2BK123 monoubiquitin group, and H3K4me3 remains as a memory mark

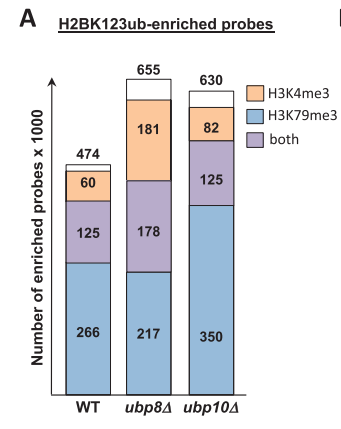

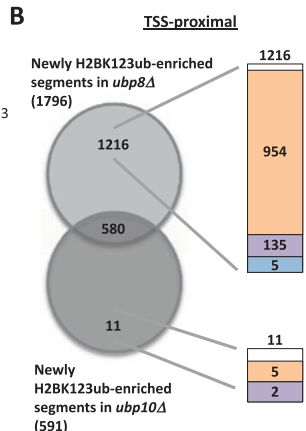

(591) might help Ubp8 to be recruited to H3K4me3-marked regions specifically (Kohler et al. 2006). Ubp10 has not been identified to be part of any complex, and the mechanism of its recruitment to chromatin remains to be determined. Although Ubp10 was proposed to play a role in telomeric silencing (Emre et al. 2005; Gardner et al. 2005), we detected no H2BK123ub in telomeric regions, including the TG repeat, $\mathrm{X}$ and $\mathrm{Y}^{\prime}$ elements in wild-type and $u b p 8$ and ubp10 deletion srains (Supplemental Fig. S5; Supplemental Table S1), and we recently demonstrated a limited role of H3K79 methylation in natural telomeric silencing (Takahashi et al. 2011). Furthermore, levels of H2BK123ub in subtelomeric regions showed minimal site-specific changes upon loss of Ubp8 or Ubp10 when compared with wild-type cells (Supplemental Fig. S5). A recent study suggests that Ubp10's role at subtelomeric regions is context-dependent

Figure 4. Ubp8 removed $\mathrm{H} 2 \mathrm{BK} 123 \mathrm{ub}$ at sites enriched for $\mathrm{H} 3 \mathrm{~K} 4 \mathrm{me} 3$, whereas Ubp10 acted on H3K79me3-marked regions. (A) All probes enriched for H2BK123ub within transcripts in wildtype as well as ubp 8 and ubp10 deletion strains were compared with the number of these probes enriched for H3K4me3 and H3K79me3. (B) Venn diagrams comparing the number of TSS-proximal and mid-CDS segments newly enriched for H2BK123ub in ubp 8 and $u b p 10$ deletion strains. Circles represent the overlap of segments newly enriched for H2BK123ub in ubp8 or ubp10 deletion strains. Bars indicate segments marked by H3K4me3 (light red), H3K79me3 (light blue), or both (purple). 


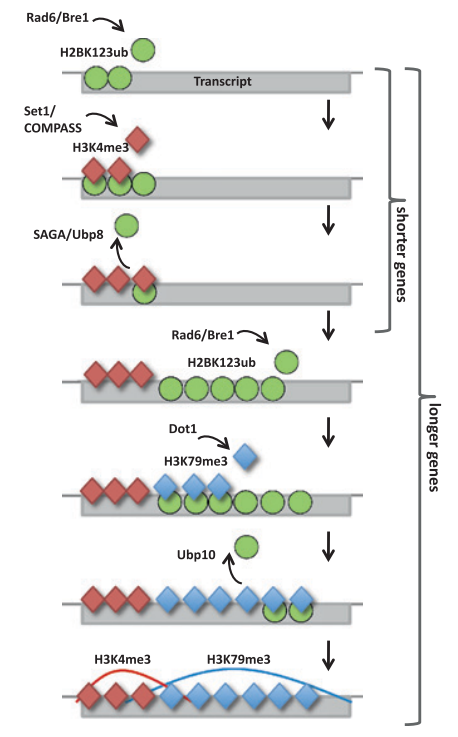

Figure 5. Model depicting the circuitry of H2BK123ub and its dependent marks, H3K4me3 and H3K79me3, in short and long genes. In the $5^{\prime}$ end of short and long genes, H2B is monoubiquitinated by Rad6/Bre1, resulting in the recruitment of Set1/COMPASS to trimethylate H3K4me3 (Shilatifard 2006). After H3K4me3 is established, Ubp8 removes H2BK123ub. In longer genes, depending on an extensive elongation phase, $\mathrm{H} 2 \mathrm{~B}$ is monoubiquitinated in the body of transcripts, which recruits Dot1 to trimethylate H3K79me3. In these regions, H2BK123ub is removed by Ubp10.

and that it only exerts its function at subtelomeres upon impairment of the H2A.Z chaperone Chzl (Wan et al. 2010). Supporting its broader role in euchromatic regions (Gardner et al. 2005), we revealed that Ubp10 specifically removes H2BK123ub from H3K79 trimethylated coding regions and provide a connection of Ubp10 (Dot4) and Dotl across the genome.

Proper addition and removal of H2BK123ub were proposed to be essential for optimal gene expression. However, impaired removal of H2BK123ub only leads to moderate effects on transcription levels (Gardner et al. 2005; Lenstra et al. 2011), and loss of Ubp8 does not alter recruitment of RNAPII to GAL1 during gene activation (Wyce et al. 2007). Here, we confirm and extend these observations by showing that the genome-wide localization of the Rpb3 subunit of RNAPII was modestly affected by loss of either Ubp8 or Ubp10 (Supplemental Fig. S6). Furthermore, the distribution of the key elongation mark H3K36me3 was not altered upon loss of Ubp8 (Supplemental Fig. S4), despite the proposed function of H2B monoubiquitination in stimulating the rate of transcription elongation (Shukla and Bhaumik 2007; Tanny et al. 2007). Together, these findings suggest that transcription elongation still takes place and that the cell is able to sufficiently transcribe genes despite impaired removal of the ubiquitin moiety on H2B. We speculate that an indirect removal of the ubiquitin mark occurs through eviction of $\mathrm{H} 2 \mathrm{~A}$ and $\mathrm{H} 2 \mathrm{~B}$ during transcription and/or histone turnover. Also, it is possible that H2BK123ub in the body of the gene may play a role in positioning of nucleosomes in front or in the wake of the transcribing RNAPII and that the observed role of H2BK123ub in elongation could be due to its role in nucleosomal positioning. Indeed, in an accompanying study, Pugh and colleagues
(Batta et al. 2011) have uncovered a role of H2BK123ub in nucleosomal organization in genic regions.

Taken together, here we comprehensively describe the chromatin network around H2BK123ub and how it relates to both dependent marks and the transcription cycle. Most importantly, our results point toward distinct roles of Ubp8 and Ubp10 in the deubiquitination machinery of eukaryotic cells and are in agreement with previous models describing deubiquitinases as major molecular regulators (D'Andrea 2010).

\section{Materials and methods}

Details of the ChIP-on-chip experiments and the genome-wide localization analyses are described in the Supplemental Material. In short, ChIPs were performed using antibodies directed against the indicated histone modifications, with a blocking peptide being added for the experiments interrogating H2BK123ub. Precipitated DNA was amplified with two rounds of T7 RNA polymerase amplification (Schulze et al. 2009) and hybridized to Affymetrix 1.0R S. cerevisiae tiling microarrays. An adapted version of the model-based analysis of tiling arrays (MAT) algorithm (Droit et al. 2010) was used to reliably detect enriched regions as described previously (Schulze et al. 2009), using mostly input DNA for normalization. In the case of $\mathrm{H} 2 \mathrm{BK} 123 \mathrm{ub}$, data were normalized to profiles obtained under identical conditions from a H2BK123A mutant yeast strain. Customwritten scripts were developed and used for subsequent data analysis as described in the Supplemental Material.

\section{Accession numbers}

Data files may be accessed online at http://www.ebi.ac.uk/arrayexpress under the accession number E-MEXP-3217.

\section{Acknowledgments}

We thank Alice Wang, Grace Leung, Edwin Smith, and Alexander Garrett for critical reading of the manuscript. Furthermore, we thank Hunter Fraser for help with the statistical analysis, Harm van Bakel for providing the detailed ChIP-on-chip protocol as well as the TSS list, and Frank Holstege for helpful discussions. J.M.S. was supported by a fellowship from the Child and Family Research Institute, and S.N. is a Leukemia and Lymphoma Society fellow. M.S.K. is a Scholar of the Michael Smith Foundation for Health Research, the Canadian Institute for Advanced Research, and the Mowafaghian Foundation. Research for this study in the laboratory of M.S.K. was supported by an operating grant from the Canadian Institute of Health Research (MOP-79442). Studies in the Shilatifard laboratory were supported by a fund provided by the National Institutes of Health (R01GM069905) to A.S.

\section{References}

Batta K, Zhang Z, Yen K, Goffman DB, Pugh BF. 2011. Genome-wide function of $\mathrm{H} 2 \mathrm{~B}$ ubiquitylation in promoter and genic regions. Genes Dev (this issue). doi: 10.1101/gad.177238.111.

D'Andrea AD. 2010. Susceptibility pathways in Fanconi's anemia and breast cancer. $N$ Engl J Med 362: 1909-1919.

Daniel JA, Torok MS, Sun Z-W, Schieltz D, Allis CD, Yates JR, Grant PA. 2004. Deubiquitination of histone H2B by a yeast acetyltransferase complex regulates transcription. J Biol Chem 279: 1867-1871.

Dover J, Schneider J, Tawiah-Boateng M, Wood A, Dean K, Johnston M, Shilatifard A. 2002. Methylation of histone H3 by COMPASS requires ubiquitination of histone H2B by Rad6. J Biol Chem 277: 28368-28371.

Droit A, Cheung C, Gottardo R. 2010. rMAT-an R/Bioconductor package for analyzing ChIP-chip experiments. Bioinformatics 26: 678-679.

Emre NCT, Ingvarsdottir K, Wyce A, Wood A, Krogan NJ, Henry KW, Li K, Marmorstein R, Greenblatt JF, Shilatifard A, et al. 2005. Maintenance of low histone ubiquitylation by Ubp10 correlates with telomereproximal Sir2 association and gene silencing. Mol Cell 17: 585-594.

Gardner RG, Nelson ZW, Gottschling DE. 2005. Ubp10/Dot4p regulates the persistence of ubiquitinated histone $\mathrm{H} 2 \mathrm{~B}$ : distinct roles in telomeric silencing and general chromatin. Mol Cell Biol 25: 6123-6139. 
Gerber M, Shilatifard A. 2003. Transcriptional elongation by RNA polymerase II and histone methylation. J Biol Chem 278: 26303-26306.

Henry KW, Wyce A, Lo W-S, Duggan LJ, Emre NCT, Kao C-F, Pillus L, Shilatifard A, Osley MA, Berger SL. 2003. Transcriptional activation via sequential histone $\mathrm{H} 2 \mathrm{~B}$ ubiquitylation and deubiquitylation, mediated by SAGA-associated Ubp8. Genes Dev 17: 2648-2663.

Holstege F, Jennings E, Wyrick J, Lee T, Hengartner C, Green M, Golub T, Lander E, Young R. 1998. Dissecting the regulatory circuitry of a eukaryotic genome. Cell 95: 717-728.

Kahana A, Gottschling DE. 1999. DOT4 links silencing and cell growth in Saccharomyces cerevisiae. Mol Cell Biol 19: 6608-6620.

Kao CF, Hillyer C, Tsukuda T, Henry K, Berger S, Osley MA. 2004. Rad6 plays a role in transcriptional activation through ubiquitylation of histone H2B. Genes Dev 18: 184-195.

Kim J, Guermah M, McGinty RK, Lee JS, Tang Z, Milne TA, Shilatifard A, Muir TW, Roeder RG. 2009. RAD6-mediated transcription-coupled H2B ubiquitylation directly stimulates H3K4 methylation in human cells. Cell 137: 459-471.

Kohler A, Pascual-Garcia P, Llopis A, Zapater M, Posas F, Hurt E, Rodriguez-Navarro S. 2006. The mRNA export factor Sus1 is involved in Spt/Ada/Gcn5 acetyltransferase-mediated H2B deubiquitinylation through its interaction with Ubp8 and Sgf11. Mol Biol Cell 17: 42284236.

Kohler A, Zimmerman E, Schneider M, Hurt E, Zheng N. 2010. Structural basis for assembly and activation of the heterotetrameric SAGA histone H2B deubiquitinase module. Cell 141: 606-617.

Krogan NJ, Dover J, Wood A, Schneider J, Heidt J, Boateng MA, Dean K, Ryan OW, Golshani A, Johnston M, et al. 2003. The Pafl complex is required for histone H3 methylation by COMPASS and Dotlp: linking transcriptional elongation to histone methylation. Mol Cell 11: 721-729.

Lee J, Shukla A, Schneider J, Swanson S, Washburn M, Florens L, Bhaumik S, Shilatifard A. 2007. Histone crosstalk between H2B monoubiquitination and H3 methylation mediated by COMPASS. Cell 131: 1084-1096.

Lee JS, Smith E, Shilatifard A. 2010. The language of histone crosstalk. Cell 142: 682-685.

Lenstra TL, Benschop JJ, Kim T, Schulze JM, Brabers NA, Margaritis T, van de Pasch LA, van Heesch SA, Brok MO, Groot Koerkamp MJ, et al. 2011. The specificity and topology of chromatin interaction pathways in yeast. Mol Cell 42: 536-549.

Liu CL, Kaplan T, Kim M, Buratowski S, Schreiber SL, Friedman N, Rando OJ. 2005. Single-nucleosome mapping of histone modifications in S. cerevisiae. PLoS Biol 3: e328. doi: 10.1371/jounral.pbio.0030328.

Mayer A, Lidschreiber M, Siebert M, Leike K, Soding J, Cramer P. 2010. Uniform transitions of the general RNA polymerase II transcription complex. Nat Struct Mol Biol 17: 1272-1278.

McGinty RK, Kim J, Chatterjee C, Roeder RG, Muir TW. 2008. Chemically ubiquitylated histone $\mathrm{H} 2 \mathrm{~B}$ stimulates hDot $1 \mathrm{~L}-\mathrm{mediated}$ intranucleosomal methylation. Nature 453: 812-816.

Minsky N, Shema E, Field Y, Schuster M, Segal E, Oren M. 2008. Monoubiquitinated $\mathrm{H} 2 \mathrm{~B}$ is associated with the transcribed region of highly expressed genes in human cells. Nat Cell Biol 10: 483-488.

Muramoto T, Müller I, Thomas G, Melvin A, Chubb JR. 2010. Methylation of $\mathrm{H} 3 \mathrm{~K} 4$ is required for inheritance of active transcriptional states. Curr Biol 20: 397-406.

$\mathrm{Ng} \mathrm{HH}$, Robert F, Young RA, Struhl K. 2003. Targeted recruitment of Setl histone methylase by elongating Pol II provides a localized mark and memory of recent transcriptional activity. Mol Cell 11: 709-719.

Samara NL, Datta AB, Berndsen CE, Zhang $X$, Yao $T$, Cohen RE, Wolberger C. 2010. Structural insights into the assembly and function of the SAGA deubiquitinating module. Science 328: 1025-1029.

Santos-Rosa H, Schneider R, Bannister AJ, Sherriff J, Bernstein BE, Emre NCT, Schreiber SL, Mellor J, Kouzarides T. 2002. Active genes are trimethylated at K4 of histone H3. Nature 419: 407-411.

Schulze J, Jackson J, Nakanishi S, Gardner J, Hentrich T, Haug J, Johnston M, Jaspersen S, Kobor M, Shilatifard A. 2009. Linking cell cycle to histone modifications: SBF and H2B monoubiquitination machinery and cellcycle regulation of H3K79 dimethylation. Mol Cell 35: 626-641.

Shilatifard A. 2006. Chromatin modifications by methylation and ubiquitination: implications in the regulation of gene expression. Annu Rev Biochem 75: 243-269.

Shukla A, Bhaumik SR. 2007. H2B-K123 ubiquitination stimulates RNAPI elongation independent of H3-K4 methylation. Biochem Biophys Res Commun 359: 214-220.
Smith E, Shilatifard A. 2009. Developmental biology. Histone cross-talk in stem cells. Science 323: 221-222.

Smith E, Shilatifard A. 2010. The chromatin signaling pathway: diverse mechanisms of recruitment of histone-modifying enzymes and varied biological outcomes. Mol Cell 40: 689-701.

Song YH, Ahn SH. 2010. A Brel-associated protein, large 1 (Lge1), promotes $\mathrm{H} 2 \mathrm{~B}$ ubiquitylation during the early stages of transcription elongation. J Biol Chem 285: 2361-2367.

Takahashi YH, Schulze JM, Jackson J, Hentrich T, Seidel C, Jaspersen SL, Kobor MS, Shilatifard A. 2011. Dot1 and histone H3K79 methylation in natural telomeric and HM silencing. Mol Cell 42: 118-126.

Tanny JC, Erdjument-Bromage H, Tempst P, Allis CD. 2007. Ubiquitylation of histone $\mathrm{H} 2 \mathrm{~B}$ controls RNA polymerase II transcription elongation independently of histone H3 methylation. Genes Dev 21: 835-847.

Wan Y, Chiang JH, Lin CH, Arens CE, Saleem RA, Smith JJ, Aitchison JD. 2010. Histone chaperone Chzlp regulates H2B ubiquitination and subtelomeric anti-silencing. Nucleic Acids Res 38: 1431-1440.

Wood A, Krogan N, Dover J, Schneider J, Heidt I, Boateng M, Dean K, Golshani A, Zhang Y, Greenblatt J, et al. 2003. Bre1, an E3 ubiquitin ligase required for recruitment and substrate selection of Rad6 at a promoter. Mol Cell 11: 267-274.

Wyce A, Xiao T, Whelan KA, Kosman C, Walter W, Eick D, Hughes TR, Krogan NJ, Strahl BD, Berger SL. 2007. H2B ubiquitylation acts as a barrier to Ctk1 nucleosomal recruitment prior to removal by Ubp8 within a SAGA-related complex. Mol Cell 27: 275-288.

Xiao T, Kao CF, Krogan NJ, Sun ZW, Greenblatt JF, Osley MA, Strahl BD. 2005. Histone $\mathrm{H} 2 \mathrm{~B}$ ubiquitylation is associated with elongating RNA polymerase II. Mol Cell Biol 25: 637-651.

Zhang L, Ma H, Pugh BF. 2011. Stable and dynamic nucleosome states during a meiotic developmental process. Genome Res 21: 875-884.

Zheng S, Wyrick IJ, Reese JC. 2010. Novel trans-tail regulation of H2B ubiquitylation and $\mathrm{H} 3 \mathrm{~K} 4$ methylation by the $\mathrm{N}$ terminus of histone H2A. Mol Cell Biol 30: 3635-3645. 


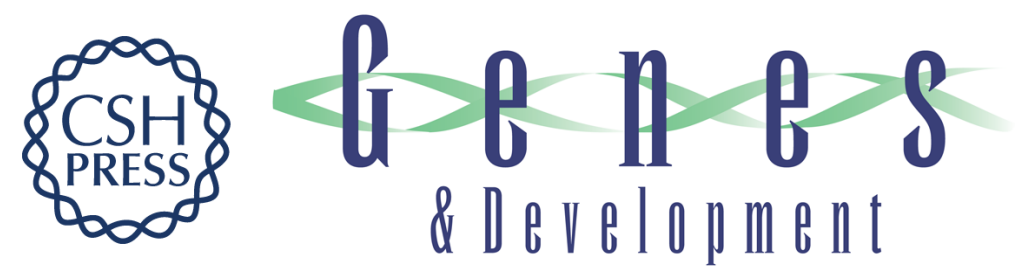

\section{Splitting the task: Ubp8 and Ubp10 deubiquitinate different cellular pools of H2BK123}

Julia M. Schulze, Thomas Hentrich, Shima Nakanishi, et al.

Genes Dev. 2011, 25:

Access the most recent version at doi:10.1101/gad.177220.111

Supplemental
Material http://genesdev.cshlp.org/content/suppl/2011/11/04/25.21.2242.DC1

References This article cites 40 articles, 15 of which can be accessed free at:

http://genesdev.cshlp.org/content/25/21/2242.full.html\#ref-list-1

License

Email Alerting Receive free email alerts when new articles cite this article - sign up in the box at the top

Service right corner of the article or click here.

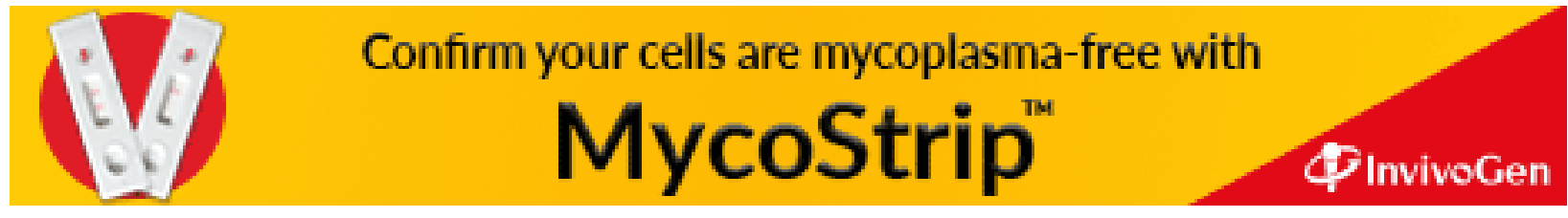

